\title{
ON THE CONTINUATION OF SOLUTIONS OF DIFFERENTIAL EQUATIONS BY VECTOR LJAPUNOV FUNCTIONS
}

\author{
L. HATVANI
}

\begin{abstract}
In this note we prove a continuation theorem applicable also when the estimate of the derivative of the vector Ljapunov function contains the phase coordinates explicitly. Our theorem combines and strengthens several earlier continuation results including a recent theorem of T. A. Burton, who conjectured that the monotonicity assumption on a function in his theorem may be dropped. By an example we show, however, that Burton's theorem would be false without this assumption. Furthermore, applying our theorem we can replace this assumption by a much weaker one.
\end{abstract}

1. Introduction. Consider the system of ordinary differential equations

$$
x^{\prime}=f(t, x),
$$

where $t \in R_{+}=[0, \infty), x=\left(x_{1}, x_{2}, \ldots, x_{n}\right) \in R^{n}$, and $f: R_{+} \times R^{n} \rightarrow R^{n}$ is continuous. Then every solution $x(t)$ is defined on a right-maximal interval $\left[t_{0}, T^{*}\right)$ $\left(t_{0}<T^{*} \leqslant \infty\right)$. Recently T. A. Burton [1] found the following result yielding the existence of each solution of (1) for all future time.

TheOREM A. Let the functions $V, g: R_{+} \times R^{n} \rightarrow R_{+} ; r: R_{+} \times R_{+} \rightarrow R ; h, \eta$ : $R_{+} \rightarrow R_{+}$and $\omega: R_{+} \rightarrow(0, \infty)$ be continuous having the following properties:

(a) for each $u_{0}>0$ the maximal solution of $\left[u^{\prime}=r(t, u), u\left(t_{0}\right)=u_{0}\right]$ exists for all $t>t_{0}$

(b) $V$ locally Lipschitz in $x$, and its derivative $V^{\prime}(t, x)$ with respect to system (1) (see [2, p. 131]) satisfies the inequality $V^{\prime}(t, x) \leqslant-g(t, x)+r(t, V(t, x))$ on $R_{+} \times$ $R^{n}$;

(c) $\omega$ is monotone nondecreasing, and $\int_{0}^{\infty}[d s / \omega(s)]=\infty$.

Suppose that for each $T>0$ and $i(1 \leqslant i \leqslant n)$ if

(A) $\left|f_{i}(t, x)\right| \leqslant \eta(t)[g(t, x)+h(V(t, x))+\omega(|x|)]$ for $0<t<T$ and $x \in R^{n}$ fails, then for each $K>0$ there exists $M>0$ such that either

(B) $V(t, x) \leqslant K, 0 \leqslant t \leqslant T$, and $x \in R^{n}$ implies $\left|f_{i}(t, x)\right|<M$, or

(C) $V(t, x) \leqslant K$ and $0 \leqslant t \leqslant T$ implies $\left|x_{i}\right| \leqslant M$ holds.

Then each solution of (1) exists for all future time.

This theorem combines the Wintner-Conti theorem (in which the existence of $V$ is not supposed, and condition (A) is satisfied for every $i$ with $g \equiv h \equiv 0$ but

Received by the editors October 6, 1978 and, in revised form, January 22, 1979.

AMS (MOS) subject classifications (1970). Primary 34A16, 34A40.

Key words and phrases. Continuation of solutions, vector Ljapunov function, differential inequalities, comparison method. 
without the monotonicity property of $\omega$ ) and the Yoshizawa theorem (in which conditions (a) and (b) are supposed with $g \equiv 0$, and $V(t, x) \rightarrow \infty$ as $x \rightarrow \infty$, uniformly for $t$ in any compact set). So Theorem $A$ gives a very flexible method to obtain continuation results, as is illustrated in [1] by interesting applications to second and third order equations. It is remarkable, however, that the monotonicity assumption on $\omega$ is not supposed in the Wintner-Conti theorem. Burton says in [1]: "Efforts to prove Theorem A without the monotonicity assumption on $\omega$ have failed, but it seems reasonable that the condition is not needed."

In this note we give an example showing that the monotonicity assumption on $\omega$ is necessary in the sense that it cannot be omitted without changing the other conditions of the theorem. But it actually seems reasonable that the assumption can be weakened essentially. Studying this problem, we establish a general continuation result for (1) by the method of vector Ljapunov functions that gives the possibility of a simple unified treatment of the methods comprised in the above mentioned theorems, and also points out the possibility of their further development.

2. A counterexample for Burton's conjecture. Let $\omega_{0}: R_{+} \rightarrow(0, \infty)$ be a continuous function with the property

$$
\int_{0}^{\infty}\left[1 / \omega_{0}(s)\right] d s=\infty ; \quad \int_{0}^{\infty}\left[1 /\left(1+\omega_{0}(s)\right)\right] d s<\infty
$$

(There exist functions having property (2), e.g., let

$$
\omega_{0}(u)= \begin{cases}(2-1 / 8)^{2} & (0 \leqslant u \leqslant 2-1 / 8) \\ 1 / k & \left(k \leqslant u \leqslant k+1 / k^{2}\right) \\ u^{2} & \left(k+1 / k^{2}+1 / k^{3} \leqslant u \leqslant(k+1)-1 /(k+1)^{3}\right) \\ & (k=2,3, \ldots) \\ \text { it is linear on the intervals left out. })\end{cases}
$$

Now put $V \equiv h \equiv 0, g \equiv r \equiv \eta \equiv 1$ and $\omega \equiv \omega_{0}$. Then it is easy to see that all the conditions of Theorem $A$ are satisfied for the scalar equation $x^{\prime}=1+\omega_{0}(|x|)$ except the monotonicity condition on $\omega$ (property (2) excludes the monotonicity of $\left.\omega_{0}\right)$. At the same time, there is no solution of this equation defined for all future time. This is obvious according to (2).

Thus, the monotonicity assumption on $\omega$ cannot be omitted from Theorem A.

3. A continuation result. According to [2], a function $W: R^{k} \rightarrow R^{k}$ is said to be quasimonotone nondecreasing if $W_{j}$ is nondecreasing in $u_{1}, u_{2}, \ldots, u_{j-1}$, $u_{j+1}, \ldots, u_{k}(j=1,2, \ldots, k)$.

Theorem. Suppose that the functions $\mathfrak{B}: R_{+} \times R^{n} \rightarrow R^{k}, w: R_{+} \times R^{n} \times R^{k} \rightarrow$ $R^{k}$ are continuous, $\mathfrak{B}(t, x)$ is locally Lipschitzian in $x, w(t, x, u)$ is quasimonotone nondecreasing with respect to $u$, and

$$
\mathfrak{B}^{\prime}(t, x) \leqslant w(t, x, \mathfrak{B}(t, x)) \quad\left((t, x) \in R_{+} \times R^{n}\right) .
$$


Let $x(t)$ be a solution of $(1)$ on $\left[t_{0}, T\right)\left(t_{0}<T<\infty\right)$. If the maximal solution $u^{*}(t)$ of the initial value problem

$$
u^{\prime}=w(t, x(t), u), \quad u\left(t_{0}\right)=\mathfrak{B}\left(t_{0}, x\left(t_{0}\right)\right)
$$

is defined on $\left[t_{0}, T\right)$, the limit from the left $u^{*}(T-0)$ exists and is finite; furthermore, if

$$
\liminf _{(t, x) \rightarrow(T-0, \infty)} p(\mathfrak{B}(t, x))>p\left(u^{*}(T-0)\right)
$$

with some monotone nondecreasing functional $p: R^{k} \rightarrow R$, then $x(t)$ is continuable to $t=T$.

Proof. Suppose that the statement is false. Then $|x(T-0)|=\infty$ (see $[2, \mathrm{p} .5])$. By (3), the function $\mathfrak{b}(t)=\mathfrak{B}(t, x(t))$ satisfies the inequality $\mathfrak{b}^{\prime}(t) \leqslant w(t, x(t), \mathfrak{b}(t))$ on $\left[t_{0}, T\right)$. Using the basic theorem of differential inequalities [2, p. 28] we get $\mathfrak{b}(t) \leqslant u^{*}(t)\left(t \in\left[t_{0}, T\right)\right)$, i.e.

$$
\liminf _{(t, x) \rightarrow(T-0, \infty)} p(\mathfrak{B}(t, x)) \leqslant \limsup _{t \rightarrow T-0} p(\mathfrak{b}(t)) \leqslant p\left(u^{*}(T-0)\right),
$$

which contradicts (5).

The theorem is proved.

REMARK 1. If we have an estimate of the type $(t, x(t)) \in D \subset\left[t_{0}, T\right) \times R^{n}$, it is sufficient to require assumption (5) only on the set $(t, x) \in D$.

REMARK 2. Essentially the proof of the theorem consists in showing the impossibility of $|x(T-0)|=\infty$. So it is easy to see that $|x| \geqslant \rho$ and $|x(t)| \geqslant \rho(t \in$ $\left.\left[t_{0}, T\right)\right)$ may be supposed in (3) and (4), respectively.

4. Applications. (A) Theorem A can be derived from our theorem in the following way. Suppose that the assumptions of Theorem A are satisfied, and let $T$ be fixed. Without loss of generality we may assume that assumptions (A), (B) and (C) are satisfied for $i=1,2, \ldots, n_{1}, i=n_{1}+1, \ldots, n_{1}+n_{2}$, and $i=n_{1}+n_{2}+$ $1, \ldots, n_{1}+n_{2}+n_{3}=n$, respectively $\left(n_{j} \geqslant 0(j=1,2,3)\right)$. Partition the vector $x \in R^{n}$ as $x=\left(x^{1}, x^{2}, x^{3}\right)\left(x^{j} \in R^{n_{j}} ; j=1,2,3\right)$, and define the auxiliary function $\mathfrak{B}$ as follows: $\mathfrak{B}(t, x)=\left(\left|x^{1}\right|,\left|x^{2}\right|, V(t, x)\right)$. A simple calculation shows that in this case the system of differential equations in (4) may be chosen to be

$$
\begin{aligned}
& u_{1}^{\prime}=n_{1} \eta(t)\left[g(t, x(t))+h(V(t, x(t)))+\omega\left(\left|\left(u_{1}, u_{2},\left|x^{3}(t)\right|\right)\right|\right)\right], \\
& u_{2}^{\prime}=\left|f^{2}(t, x(t))\right|, \quad u_{3}^{\prime}=-g(t, x(t))+r\left(t, u_{3}\right) .
\end{aligned}
$$

Let us prove that the assumptions of our theorem are satisfied. By assumption (a) in Theorem $\mathrm{A}, u_{3}(t)$ is bounded on $\left[t_{0}, T\right)$ and thus $V(t, x(t))$ is bounded there, too. By virtue of (B) and (C), this implies that $u_{2}(t)$ and $\left|x^{3}(t)\right|$ are bounded on $\left[t_{1}, T\right)$. By the last equation of $(6), g(t, x(t))$ is integrable on $\left[t_{0}, T\right)$; hence there are $a, b \geqslant 0$ such that

$$
u_{1}(t) \leqslant a+n_{1} \int_{t_{0}}^{t} \eta(s) \omega\left(u_{1}(s)+b\right) d s \quad\left(t_{0} \leqslant t<T\right) .
$$

By Bihari's lemma [2, p. 39] it follows that $u_{1}(t)$ also bounded on $\left[t_{0}, T\right)$; therefore, the limit $u(T-0)$ exists and is finite. Since $\lim _{x \rightarrow \infty}|\mathfrak{B}(t, x)|=\infty$, assumption (5) is satisfied with $p(u)=|u|$. 
(B) The example in $\$ 2$ shows that the monotonicity assumption on $\omega$ cannot be omitted from Theorem A. Now it will be proved, however, that the only role of the monotonicity of $\omega$ is to exclude the possibility of (2).

Replace assumption (A) in Theorem $\mathrm{A}$ by

$$
\left|f_{i}(t, x)\right| \leqslant \eta(t)\left[g(t, x)+h(V(t, x))+\omega\left(\left|x^{1}\right|\right)\right]
$$

for $i=1,2, \ldots, n_{1}$, where $\omega$ is not supposed to be monotone (as for the notations $x^{1}, n_{1}$, see $\left.\S 4,(\mathrm{~A})\right)$, and define the auxiliary functions $\mathfrak{B}, w$ as follows:

$$
\begin{gathered}
\mathfrak{B}=\left(n_{1} \int_{t}^{T} \eta(s) d s+\int_{0}^{\left|x^{2}\right|} \frac{d z}{c+\omega(z)},\left|x^{2}\right|, V(t, x)\right) ; \\
w=\left(n_{1} \eta(t)[g(t, x(t))+h(V(t, x(t)))],\left|f^{2}(t, x(t))\right|,-g(t, x(t))+r\left(t, u_{3}\right)\right) ; \\
p(u)=|u|,
\end{gathered}
$$

where $c=0$ if $g \equiv h \equiv 0$ and $c=1$ otherwise. Similarly to example (A), $u_{2}(T-0)$ and $u_{3}(T-0)$ exist and are finite; furthermore, $g(t, x(t))$ is integrable and $h(V(t, x(t)))$ is bounded on $\left[t_{0}, T\right)$. Now $u_{1}(T-0)$ exists and is finite obviously. In order to obtain $\lim _{x \rightarrow \infty}|\mathfrak{B}(t, x)|=\infty$, we have to require

$$
\text { either } g \equiv h \equiv 0 \quad \text { or } \int_{0}^{\infty} \frac{d s}{1+\omega(s)}=\infty
$$

Therefore we can modify Theorem A replacing the monotonicity of $\omega$ and assumption (A) by assumptions (7) and ( $\left.A^{\prime}\right)$, respectively. The Wintner-Conti theorem is already a direct corollary of this modification of Theorem A ( $g \equiv h \equiv$ 0).

Not only in these examples but also in numerous applications of Ljapunov's direct method, only estimations of the type (3) are known for the derivative of the Ljapunov function, i.e. the majorizing function also contains the phase coordinates $x$ explicitly (e.g. [1], [4], [5]). In these cases, standard theorems of the comparison method [2], [3] cannot be applied directly and it is necessary to introduce comparison systems of the type (4).

The author is indebted to the referee for his constructive criticisms.

\section{REFERENCES}

1. T. A. Burton, A continuation result for differential equations, Proc. Amer. Math. Soc. 67 (1977), 272-276.

2. V. Lakshmikantham and S. Leela, Differential and integral inequalities, Vol. I, Academic Press, New York, 1969.

3. V. M. Matrosov, On the theory of stability of motion, Prikl. Mat. Meh. 26 (1962), 992-1002; English transl., J. Appl. Math. Mech. 26 (1963), 1506-1522. MR 27, 403.

4. __ On the theory of stability of motion. II, Trudy Kazan. Aviacion. Inst. 80 (1963), 22-33. MR 34, 6239. (Russian)

5. L. Hatvani, The application of differential inequalities to stability theory, Vestnik Moskov. Univ. Ser. I Math. Meh. (3) 30 (1975), 83-89. MR 52, 928. (Russian)

Bolya Institut, Aradi Vértanuk Tere 1, Szeged, Hungary 